\title{
Determination of total and available fractions of PAHs by SPME in oily wastewaters: overcoming interference from NAPL and NOM
}

\author{
Rui B. Gomes • Regina Nogueira • José M. Oliveira • \\ João Peixoto • António G. Brito
}

Received: 14 December 2007 / Accepted: 22 February 2009/Published online: 17 March 2009

(C) Springer-Verlag 2009

\begin{abstract}
Background, aim, and scope Polycyclic aromatic hydrocarbons (PAHs) are often found in oily wastewaters. Their presence is usually the result of human activities and has a negative effect on the environment. One important step in addressing this problem is to evaluate the effectiveness of PAH removal by biological processes since these are the most cost-effective treatments known today. Many techniques are presently available for $\mathrm{PAH}$ determination in wastewaters. Solid phase microextracion (SPME) is known to be one of the most effective techniques for this purpose. When analyzing complex matrices with substances such as natural organic matter (NOM) and non-aqueous phase liquids (NAPL), it is important to differentiate the free dissolved PAH from matrix-bonded PAH. PAHs associated with the bonded fraction are less susceptible to biological treatment. The present study concerns the development of a simple and suitable methodology for the determination of the freely dissolved and the total fraction of PAHs present in oily wastewaters. The methodology was then applied to an oily wastewater from a fuel station retention basin.

Material and methods Headspace SPME was used for analyzing PAH since the presence of a complex or dirty matrix in direct contact with the fiber may damage it. Four model PAHs - anthracene, fluorene, phenanthrene, and pyrene-were analyzed by GC-MS. Negligible depletion SPME technique was used to determine the free fraction.

Responsible editor: Ludek Blaha

R. B. Gomes $\cdot$ R. Nogueira $\cdot$ J. M. Oliveira $\cdot$ J. Peixoto $\cdot$

A. G. Brito $(\bowtie)$

Institute for Biotechnology and Bioengineering,

Centre of Biological Engineering, University of Minho,

Campus de Gualtar,

4710-057 Braga, Portugal

e-mail: agbrito@deb.uminho.pt
\end{abstract}

Total PAH was determined by enhancing the mass transfer from the bonded phase to the freely dissolved phase by temperature optimization and the use of the method of standard additions. The PAH absorption kinetics were determined in order to define the optimal sampling conditions for this method. The fitting of the experimental data to a mathematical model was accomplished using Berkeley Madonna software. Humic acid and silicon oil were used as model NOM and NAPL, respectively, to study the effect of these compounds on the decrease of SPME response. Then, the method was evaluated with wastewater from a fuel station spill retention basin.

Results The SPME kinetic parameters - $k_{1}$ (uptake rate), $k_{2}$ (desorption rate), and $K_{\mathrm{SPME}}$ (partition coefficient)-were determined from experimental data modeling. The determination of the free fraction required 15-min sampling to ensure that PAH depletion from sample was below $1 \%$. For total $\mathrm{PAH}$, a 30 -min extraction at $100^{\circ} \mathrm{C}$ ensured the maximum signal response in the GC-MS. For the determination of free and total PAHs, extractions were performed before reaching the SPME equilibrium. The wastewater used in this study had no free fraction of the analyzed PAHs. However, the four studied PAHs were found when the method for total PAH was used.

Discussion The addition of NOM and NAPL dramatically decreased the efficiency of the SPME. This decrease was the result of a greater partition of the PAHs to the NAPL and NOM phases. This fact was also observed in the analysis of the fuel station spill retention basin, where no free PAH was measured. However, using the method of standard addition for the determination of total PAH, it was possible to quantify all four PAHs.

Conclusions The method developed in the present study was found to be adequate to differentiate between free and total PAH present in oily wastewater. It was determined that 
the presence of NOM and NAPL had a negative effect on SPME efficiency.

Recommendations and perspectives The presence of binding substances had a great influence on SPME kinetics. Therefore, it is of extreme importance to determine their degree of interference when analyzing oily wastewaters or results can otherwise be erroneous. Other factors influencing the total PAH determinations should be considered in further studies.

Keywords Free PAHs · HS-SPME · NAPL · NOM · Oily wastewater Polycyclic aromatic hydrocarbons . Total PAH

\section{Background, aim, and scope}

Polycyclic aromatic hydrocarbons (PAHs) are environmental contaminants with toxic and carcinogenic effects. Their chemical structure is responsible for low solubility in water and poor biodegradability, and they are found in the environment at several concentrations (Samanta et al. 2002). Doong and Lin (2004) and Busetti et al. (2006) state that these concentrations depend on the level of contamination and the medium of nature (e.g., soil, sludge, natural water streams, wastewaters). Regarding to wastewaters, the PAH concentration is strongly related to the nature of the activity that produces the wastewater. Petrochemical and oil-related activities, for instance, are known to produce strong $\mathrm{PAH}$ contamination (Al Zarooni and Elshorbagy 2006). The large range of occurring PAH concentrations leads to the necessity of a good characterization of the wastewater.

The presence of binding substances may cause the bioavailability of PAH to decrease even further (Kubicki and Apitz 1999; Kose et al. 2003). Oily wastewaters may contain many substances that interfere with PAH bioavailability. They are the result of human activities and industrial processes dealing with fuels, solvents, and oils, among other things. They usually are the main source of PAH pollution in water environments.

Natural organic matter (NOM) is known to have binding effects towards PAH (Maxin and Kogel-Knabner 1995). These binding interactions can be partitioning or adsorption phenomena, depending on the nature of the organic matter (Poerschmann and Kopinke 2001). The distribution or partitioning of non-ionic hydrophobic organic compounds like PAH between water and soil or sediment has been shown to depend primarily on the hydrophobicity of the compounds and on the fraction of organic carbon in the sorbent (Schlautman and Morgan 1993). Factors like temperature, salinity, and $\mathrm{pH}$ also influence the degree of PAH binding to these substances. Schlautman and Morgan (1993) stated that the binding of PAHs to humic material, in $\mathrm{NaCl}$ solutions, generally decreases with increases in $\mathrm{pH}$ (constant ionic strength) and decreases with increasing ionic strength (fixed $\mathrm{pH}$ ).

Non-aqueous phase liquids (NAPL) are also present in oily wastewaters. They are reported to act as solvents for solid low-bioavailable PAH, thus enhancing the transfer rates of PAHs to biological agents (Lee et al. 2001). They are present in many wastewaters, including those related with oil activities (Shokrollahzadeh et al. 2008). Both NOM and NAPL are important factors to take into account when studying PAH in water environments (Kubicki and Apitz 1999; Lesage et al. 2001).

The methods used to determine the concentrations of $\mathrm{PAH}$ in complex matrices like oily wastewaters present some drawbacks. Specific techniques are required to quantify the PAH fractions freely dissolved in water and bonded to other chemical substances. Solid phase microextraction (SPME) is currently recognized as the most useful method for such kinds of determination (Poerschmann et al. 1998; Ramos et al. 1998; King et al. 2003, 2004). SPME is a technique introduced in 1990 by Arthur and Pawliszyn (1990) based on a small fused silica fiber coated with a polymeric substance. The fiber adsorbs the target compound prior to injection in a chromatographic system. The chemical interactions between the target compound and the coating material depend on the nature of the material. A solid polymer such as polyacrylate extracts the compound by adsorption. Liquid polymers (e.g., polydimethylsiloxane [PDMS]), absorb the compound, presenting a partitioning equilibrium between fiber and matrix (Mayer et al. 2000). This technique is explained in detail in Pawliszyn (1997). Recent developments in SPME have enabled the use of this technique for better analysis of many different pollutants in environmental samples (Ouyang and Pawliszyn 2006a, b). SPME has several advantages over other analytical techniques such as solvent extraction since it is solvent free, faster, and more selective. PDMS fibers are the most commonly used in the determination of hydrophobic compounds like PAHs (Arthur et al. 1992; Potter and Pawliszyn 1994). The extraction implies a partitioning equilibrium between the fiber and the liquid matrix in the case of a direct immersion SPME and between fiber, matrix, and headspace in the case of headspace (HS) SPME. HSSPME is often used when dealing with solid matrices such as soil and sediments (Zhang and Pawliszyn 1993c) and complex or dirty liquid matrices such as oily wastewaters (Van Hamme and Ward 2000; Luan et al. 2007). This method involves the mass transfer of the compound from the matrix to the headspace and subsequently to the fiber (Zhang and Pawliszyn 1993b), until the equilibrium state is reached. Equilibrium is reached between the three phases, and the presence of a headspace avoids the direct contact with the dirty sample that could damage the fiber.

In the case of water environments where both NOM and NAPL are present, HS-SPME is the most adequate method 
for PAH determination, for the reasons explained earlier. However, it is important to address the nature of the PAH that is being determined as to whether it involves the free or the bonded fraction. Thus, certain modifications in the methods are needed to differentiate between free and total amount. Analyte uptake by the fiber must be negligible to determine the fraction of free PAH (Gorecki and Pawliszyn 1997). In negligible depletion (ND) SPME, the amount of analyte uptake by the fiber is small when compared to the freely dissolved amount $(\leq 1 \%)$. Therefore, the partition equilibrium state between fiber and sample is not disturbed. Thus, knowledge of equilibrium kinetics is needed to determine the optimum extraction time.

It is necessary to shift the equilibrium towards the freely dissolved fraction to determine the total amount of PAH in a complex matrix sample. This could be achieved by applying changes in a multitude of parameters like temperature, $\mathrm{pH}$, salt concentration, and surfactant addition (Pawliszyn 1997; Zhang and Pawliszyn 1993a). Temperature is thought to be one of the most influential parameters in increasing the free dissolved fraction (Zhang and Pawliszyn 1993b). This knowledge has been successfully used to extract PAH from complex matrices (Doong et al. 2000). Therefore, an increase in temperature should shift the equilibrium from the matrix phase to the free dissolved phase.

The work presented here aimed to define a suitable, simple, and useful methodology to determine the nature of PAH present in an oily wastewater. Partition kinetics was determined to define optimal conditions for SMPE and NDSPME. Model humic acid and silicon oil were used to assess their direct and combined effect on PAH determination. PAHs were used in a range of concentration covering the water solubility levels. The freely dissolved and total PAHs were determined in a sample of an oily wastewater from the retention basin of a fuel station.

\section{Materials and methods}

\subsection{Chemicals}

Fluorene (98\%) and pyrene (98\%) were purchased from Sigma-Aldrich, Germany; phenanthrene (98\%), anthracene (99\%), and acenaphtene d-10 from Sigma-Aldrich, USA; ethanol (absolute) from Merck, Germany; silicon oil AP 150 Wacker from Fluka, Germany; and humic acids from Fluka, Switzerland.

\subsection{SPME general procedure}

HS-SPME extractions were performed in $15-\mathrm{mL}$ amber glass vials (18-mm internal diameter) sealed with rubber septum coated internally with PTFE. A $100-\mu \mathrm{m}$ film thickness PDMS fiber was used. All equipment, including SMPE manual holder, were purchased from Supelco (Germany). Aqueous samples $(5 \mathrm{~mL})$ containing four model PAHs - anthracene, fluorene, phenanthrene, and pyrene-were used. The headspace to sample ratio was 3 . Acenaphthene d-10 was used as an internal standard for all calibrations.

The fiber was conditioned at the injection port at $250^{\circ} \mathrm{C}$ for $1 \mathrm{~h}$, according to the manufacturer specifications. For all extractions, the vials were stabilized for $10 \mathrm{~min}$ at the target temperature, before piercing the rubber septum with the SPME needle. Extractions were carried out at maximum magnetic stirring $\left(10,000 \mathrm{~min}^{-1}\right)$. After extraction, the fiber was placed in the CG-MS injector for $15 \mathrm{~min}$ for total desorption. Each extraction was performed in triplicate.

\subsection{SPME kinetic parameters and free PAH determination}

Four model PAHs solutions were used at $50 \%$ of their water solubility at $20^{\circ} \mathrm{C}: 66 \mu \mathrm{g} \mathrm{L}^{-1}$ for anthracene, $1,998 \mu \mathrm{g} \mathrm{L}^{-1}$ for fluorene, $1,283 \mu \mathrm{g} \mathrm{L}^{-1}$ for phenanthrene, and $146 \mu \mathrm{g} \mathrm{L}^{-1}$ for pyrene (Rumble et al. 2001). PAHs were measured after 5-, 10-, 20-, 30-, 40-, and 50-min extraction times, at $20^{\circ} \mathrm{C}$, using the general procedure referred to under Section 2.2.

Considering the SPME to be a one-compartment system and first-order kinetics, the PAH concentration in the fiber (Vaes et al. 1996) is given by

$\frac{\mathrm{d}[X]_{\mathrm{SPME}}}{\mathrm{d} t}=k_{1}[X]_{\mathrm{a}}-k_{2}[X]_{\mathrm{SPME}}$

where $[X]_{\text {SPME }}$ is the PAH concentration in the fiber, $[X]_{\mathrm{a}}$ is the aqueous PAH concentration, and $k_{1}$ and $k_{2}$ are the uptake and elimination rates, respectively. The partition coefficient is given by

$K_{\mathrm{SPME}}=\frac{[X]_{\mathrm{SPME}}}{[X]_{\mathrm{a}}}=\frac{k_{1}}{k_{2}}$

This model is suited for direct immersion SPME. However, it can also be applied to HS-SPME considering a partition between the fiber and the sample/headspace system. In this case, $[X]_{\mathrm{a}}$ is the concentration in the aqueous/headspace phase, $k_{1}$ is the uptake from the aqueous/headspace to the fiber, and $k_{2}$ is the elimination rate from the fiber to the aqueous/headspace. $K_{\mathrm{SPME}}$ becomes the partition coefficient between the fiber and the aqueous/headspace system.

For the determination of the freely dissolved fraction, the amount extracted must be less than $1 \%$ in order to not disturb the partition equilibrium. As explained by Ai (1997) 
and Bartelt and Zilkowski (1999), a quantitative analysis can be carried out before reaching partition equilibrium. When performing SPME manual sampling, an experimental uncertainty is associated. To ensure that this uncertainty does not compromise the method, Vaes et al. (1996) stipulated that a 1-min sampling variation should not lead to a deviation in the concentration by more than $5 \%$, as described by

$\frac{\mathrm{d}[X]_{\mathrm{SPME}}}{\mathrm{d} t} \leq 0.05[X]_{\mathrm{SPME}}$

The combination of Eq. 3 with an integrated form of Eq. 1 yields

$t_{\mathrm{s}} \geq-\frac{1}{k_{2}} \ln \left(\frac{0.05}{k_{2}+0.05}\right)$

where $t_{\mathrm{s}}$ is the minimum sampling time.

The method used in this study consists of the quantification of the time necessary to reach an amount of the analyte in the fiber that is $1 \%$ of the initial amount in the sample. Determining the kinetic parameters in Eq. 1 allows for the estimation of the optimal extraction time for NDSPME.

Depletion was calculated as

$\frac{\left[X_{\mathrm{SPME}}\right] V_{\mathrm{f}}}{\left[X_{\mathrm{a}}\right] V_{\mathrm{a}}} \times 100 \%$

with $V_{\mathrm{f}}$ being the fiber volume and $V_{\mathrm{a}}$ the sample volume. $[X]_{\text {SPME }}$ was calculated using the integrated version of Eq. 1. This method is suitable for the measurement of the freely available PAH and consequently also for the bioavailable PAH fraction in water samples (Poerschmann and Kopinke 2001; Ramos et al. 1998).

HS-SPME kinetics were modeled and fitted to experimental data obtained by performing extractions at different exposure times. Parameter estimation was performed using the Berkeley Madonna mathematical modeling software (Macey and Oster 1993).

After ND-SPME optimization $\left(15 \mathrm{~min}\right.$ at $\left.10^{\circ} \mathrm{C}\right)$, calibrations were performed with ultrapure water in the range of $10 \%$ to $90 \%$ of the PAH water solubility. Acenaphthene $\mathrm{d}-10$, the internal standard, was added to the standards, at a mass per volume ratio of $100 \mu \mathrm{g} \mathrm{L}^{-1}$.

\subsection{Temperature optimization and total PAH determination}

In order to optimize the PAHs measurement, five different temperatures were studied $\left(20^{\circ} \mathrm{C}, 50^{\circ} \mathrm{C}, 100^{\circ} \mathrm{C}, 150^{\circ} \mathrm{C}\right.$, and $200^{\circ} \mathrm{C}$ ) at 30 -min extraction time and PAHs concentrations of $50 \%$ of their water solubility. SPME was performed at $20^{\circ} \mathrm{C}$, in aqueous solutions. Then, for the determination of the total $\mathrm{PAH}$, the optimal temperature determined above was used to maximize desorption from the matrix. The method of standard additions was used in order to take into account the matrix effect in the total PAH determination. Five increasing quantities of $\mathrm{PAH}$, ranging from $10 \%$ to $90 \%$ of the solubility concentration, were added to the sample to be analyzed. Therefore, calibration was performed directly on the sample, allowing one to account for the matrix effect.

\subsection{GC-MS analysis}

Gas chromatographic analysis of PAH was performed using a Varian 4000 GC-MS, with a Factor Four capillary column $(30 \mathrm{~m} \times 0.25 \mathrm{~mm}, 0.25 \mu \mathrm{m}$ film thickness, Varian). The injector temperature (split/splitless) was $250^{\circ} \mathrm{C}$. The oven was set to an initial temperature of $60^{\circ} \mathrm{C}$ with an increase of $10^{\circ} \mathrm{C} \mathrm{min}$ min $^{-1}$ until a final temperature of $310^{\circ} \mathrm{C}$, followed then by a holding time of $5 \mathrm{~min}$ at the maximum temperature. The injection was carried out in split mode with an initial split ratio of 30 . Then, it was changed to 0 , at $0.001 \mathrm{~s}$, to concentrate the desorbed sample. Finally, it was set to 80 after $5 \mathrm{~min}$, for fiber cleaning purposes. The carrier gas was helium N60 (Praxair), at a flow rate of $1 \mathrm{~mL} \mathrm{~min}^{-1}$. The ion trap detector was set to electron impact mode, at $70 \mathrm{eV}$, on full scan mode, with an acquisition range $(\mathrm{m} / \mathrm{z})$ from 50 to 205 and an acquisition frequency of $2.38 \mathrm{~s}^{-1}$.

PAH identification was achieved by comparing their linear retention index and mass spectra with those of pure standards.

\subsection{Effect of model NAPL and NOM}

A factorial experimental design, with a $2 \times 2$ factor, was used to evaluate the effect of NOM and NAPL on the SPME extraction efficiency. Humic acid and silicon oil were used to represent NOM and NAPL, respectively. Both components were tested at high and low concentrations, 2 and $10 \mathrm{mg} \mathrm{L}^{-1}$ for humic acid and 20 and $100 \mathrm{~mL} \mathrm{~L}^{-1}$ for silicon oil. The values of NOM are in the concentration intervals that could be found in water environments (Wong et al. 2007; Brigante et al. 2008). The values of NAPL are also those commonly found in oily wastewaters (Zhu and Tang 2005). PAHs were used at $50 \%$ of their water solubility concentration. For each combination, a set of three determinations was used, resulting in a total of 12 extractions. The results were compared to a HS-SPME from a sample that contained only pure water. SPME conditions followed the general procedure described in Section 2.2, with 30-min sampling at $100^{\circ} \mathrm{C}$. 
Table 1 Kinetic parameters for the SPME absorption profile

\begin{tabular}{lcccccc}
\hline & $k_{1} / \min$ & $k_{2} / \min$ & $\log K_{\mathrm{SPME}}$ & $r$ & $t_{\mathrm{ND}} / \min$ & Depletion/\% \\
\hline Anthracene & 297 & 0.07 & 3.64 & 0.97 & 12.6 & 0.76 \\
Fluorene & 555 & 0.13 & 3.63 & 0.99 & 9.8 & 0.61 \\
Phenanthrene & 291 & 0.07 & 3.60 & 1.00 & 12.3 & 0.74 \\
Pyrene & 206 & 0.04 & 3.76 & 0.99 & 15.1 & 1.05 \\
\hline
\end{tabular}

\subsection{Wastewater sample}

The wastewater sample was collected from a fuel station spill retention basin. It contained both NOM and NAPL resulting from rainwater runoff and the fuel spills, respectively. Determinations were made for both freely dissolved PAH and total PAH using the methods developed in the present work. Since the wastewater contained a large number of compounds and this fact could interfere with the PAH quantification, the specific quantification ions 178 , 166, 178, and 200 were used for anthracene, fluorene, phenanthrene, and pyrene, respectively.

\section{Results and discussion}

\subsection{SPME kinetics and determination of ND-SPME conditions}

As referred to by Vaes et al. (1996), the determination of free dissolved PAHs may be conducted under ND-SPME, which serves to consider a maximum depletion of $1 \%$. In order to estimate ND-SPME best conditions, kinetic parameters were determined (Table 1) from the absorption profiles to the fiber (Fig. 1) using Berkeley Madonna software. As depicted in Section 2.3, calibrations were performed at a range of $10 \%$ to $90 \%$ of their water solubility. The obtained SPME absorption profiles were adequately described by Eq. 1, as indicated by the correlation coefficients: $r^{2}$ values were $0.98,0.95,0.94$, and 0.95 for anthracene, fluorene, phenanthrene, and pyrene, respectively. It should be noted that the uptake rate constants are much higher than the elimination rate constants. This is due to the high PAH hydrophobicity. The partition coefficient to the fiber was calculated using Eq. 2. The values found are in accordance with the partition coefficient found for hydrophobic compounds (Ramos et al. 1998; Mayer et al. 2000). Although SPME is a partition equilibrium method, a non-equilibrium determination is possible. In fact, this is necessary if the aim is to adopt NDSPME. To implement a faster and easier method, the determinations were performed before reaching partition equilibrium. As explained in Section 2.3, a minimum sampling time is needed and may be calculated with Eq. 4. The values varied from $9.8 \mathrm{~min}$ for fluorene to $15 \mathrm{~min}$ for pyrene. Therefore, 15 -min sampling time can be used as a compromise between a negligible depletion and a good accuracy for the method. Since, in ND-SPME, the
Fig. 1 Fiber absorption profiles for the four PAHs used to determine the parameters for modeling PAH uptake. PAH concentration $[P A H]$ is given as a response over the extraction time. The amount of PAH increases over time until equilibrium is reached
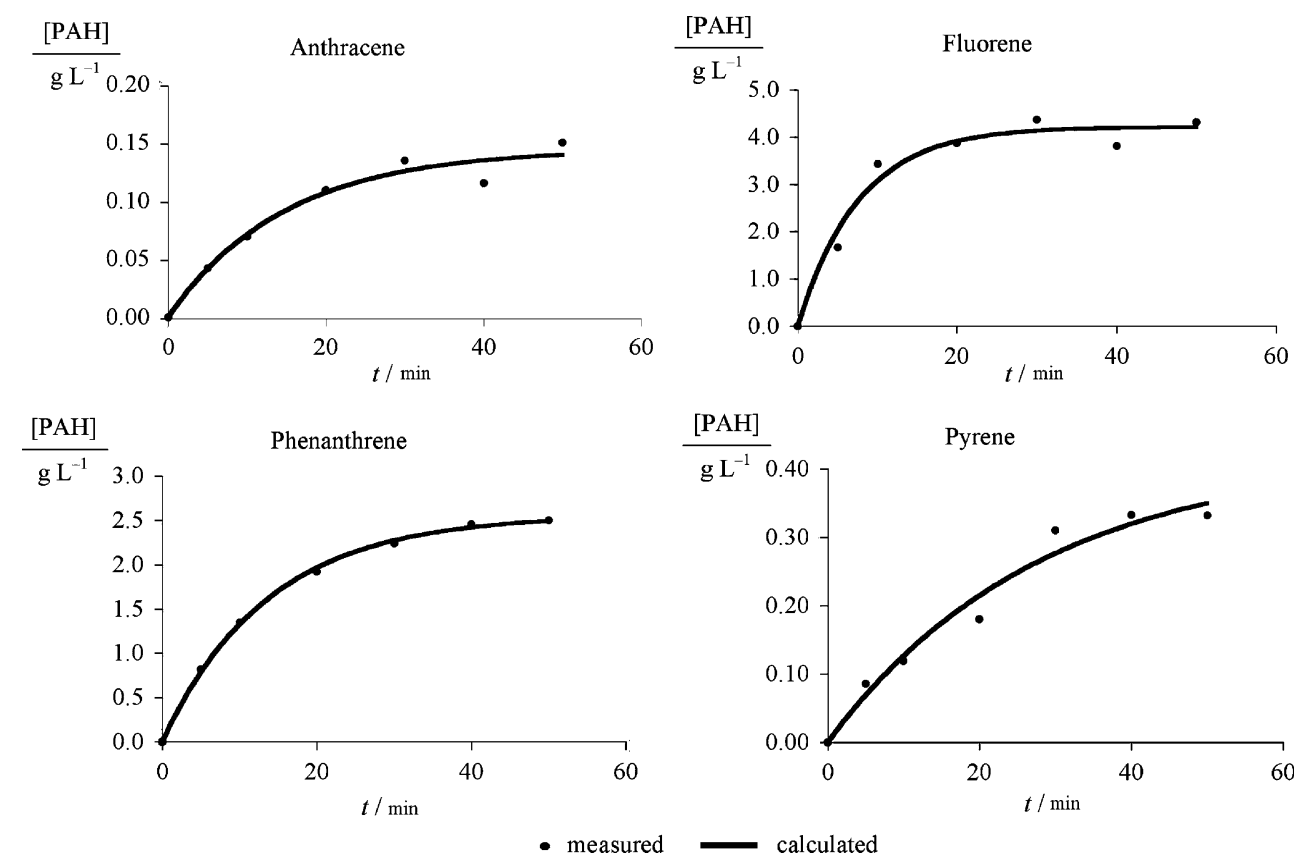
objective is not to disturb the partition equilibrium, sampling was performed at $20^{\circ} \mathrm{C}$. This temperature stands for a good mean value to represent natural (water streams) and industrial (wastewaters) samples and interior room temperatures.

\subsection{Temperature optimization for PAH extraction}

In the present study, five temperature levels were examined: $20^{\circ} \mathrm{C}, 50^{\circ} \mathrm{C}, 100^{\circ} \mathrm{C}, 150^{\circ} \mathrm{C}$, and $200^{\circ} \mathrm{C}$. A 30 -min extraction of PAHs at $50 \%$ of their solubility in water was used in aqueous solutions. The results are presented in Fig. 2. The optimal response was obtained for temperatures between $100^{\circ} \mathrm{C}$ and $150^{\circ} \mathrm{C}$. At $200^{\circ} \mathrm{C}$, all PAHs had a lower response to the extraction. This can be explained by the fact that the PAHs are desorbed from the fiber at high temperatures (Pawliszyn 1997). Pyrene showed a lower decrease at high temperatures, probably due to its lower volatility and, consequently, the need for an even higher desorption temperature (Arthur and Pawliszyn 1990).

An extraction temperature of $100^{\circ} \mathrm{C}$ was selected for the total PAH determination where an acceptable response was observed. This temperature was well below the point $\left(150^{\circ} \mathrm{C}\right)$ at which the response started to decay. Equilibrium SPME should be more accurate for total PAH determination. However, as shown earlier, the sampling time would significantly increase in this case. Therefore, a non-equilibrium extraction was performed in order to draw a time-friendly methodology. As stated earlier, for a non-equilibrium determination, a minimum sampling time is needed to ensure that no excessive deviations are present. Using a 30-min sampling, the deviations associated to the uncertainty were clearly below the minimum (Eq. 4). Therefore, this time was chosen for the extraction method.

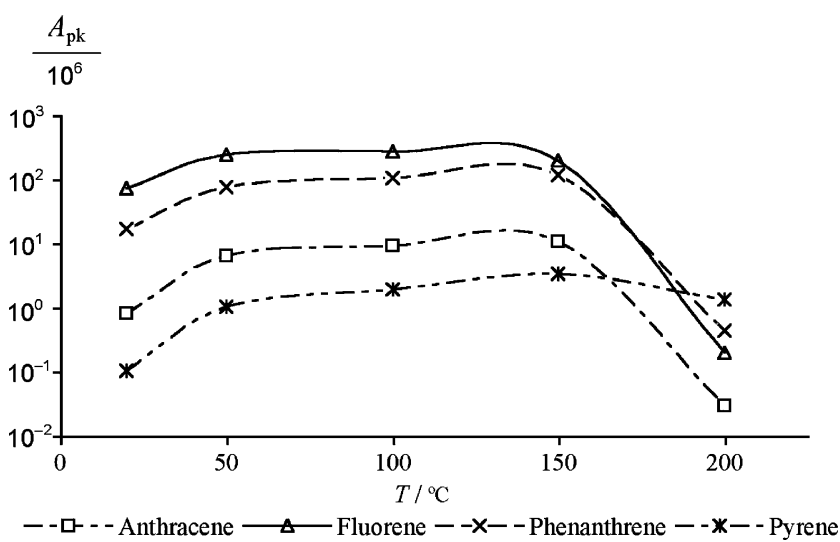

Fig. 2 Effect of temperature in PAH detection response (peak area- $A_{\mathrm{pk}}$ ) for the HS-SPME extraction. Peak area is given as a response over temperature of extraction. An optimal temperature regarding all studied PAHs is reached around $100^{\circ} \mathrm{C}$

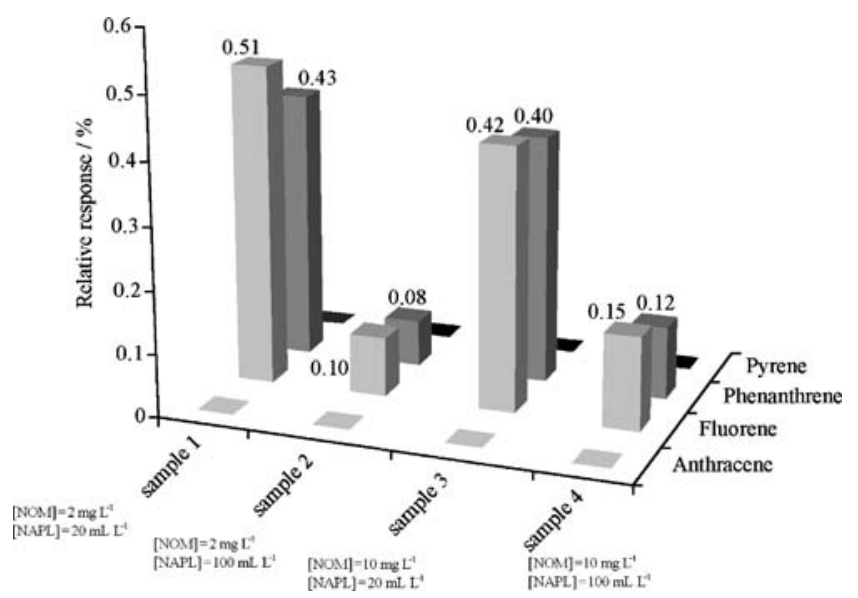

Fig. 3 PAHs measured in a sample containing a matrix of model NOM (humic acids) and NAPL (silicon oil) when compared to a sample without it. The relative response (detector response) for a sample in a complex matrix over the detector response for a sample without it

\subsection{The role of NAPL and NOM in the binding of PAHs}

According to the methodology defined under Section 2.6, it was verified that the addition of both NOM and NAPL greatly influenced the amount of PAHs in the different phases. The amount of NOM and NAPL are the ones often found in water ecosystems and wastewaters. As is shown in Fig. 3, anthracene and pyrene could not be quantified. Fluorene and phenanthrene were detected but in very low amounts. The response obtained in the presence of NOM and NAPL is always below $1 \%$ when compared to the one obtained in their absence. In contrast to ND-SPME, this indicates a very strong PAH binding to the matrix. It was observed that the increase of the amount of silicon oil in the sample leads to the major decrease of the PAH SPME detection response. However, a much broader study would be needed to clearly state which fraction, NOM or NAPL, is the major one responsible for the decrease in SPME extraction. We would probably need to use different kinds of NAPL and NOM since each one would generate dissimilar responses in the SPME extraction. So, in the current experiment, it can be said that the presence of both NAPL and NOM greatly contributes to a significant

Table 2 Quantification ions, total amount of PAHs present in the oily wastewater, and $r^{2}$ values for the calibration curves of the total PAH determination

\begin{tabular}{lccc}
\hline PAH & Quantification ion & $\frac{C_{\text {total }}}{\mu \mathrm{g} \mathrm{L}^{-1}}$ & $r^{2}$ \\
\hline Anthracene & 178 & 3.96 & 0.99 \\
Fluorene & 166 & 385 & 0.87 \\
Phenanthrene & 178 & 151 & 0.96 \\
Pyrene & 200 & 4.10 & 0.99 \\
\hline
\end{tabular}


reduction of PAH uptake in the SPME fiber. Many studies using humic acids (Ramos et al. 1998; Schlautman and Morgan 1993) reported a decrease in the response of SPME. The nature of the organic compounds is also important for the binding effects on PAHs (Jones and Tiller 1999). Therefore, care should be taken when analyzing PAH in complex matrices. The chemical composition of the matrix has a significant impact on binding. Each case should be carefully analyzed. A good strategy to overcome the matrix effect is to use a sample obtained from a contaminated site or wastewater where the matrix is present for method calibration. For example, this could be accomplished by the method of standard additions.

\subsection{PAH determination in oily wastewater and differentiation between free and total PAH}

The fuel station wastewater (see Section 2.7) was analyzed by HS-SPME for total and free dissolved PAH. Both methods for the quantification of free (see Section 2.3) and total (Section 2.4) PAHs were applied. As shown in Table 2, the four PAHs were found in the wastewater. Because oily wastewater contains high levels of contaminants that might add interferences to the chromatography, the PAHs were measured by their quantification ions. Since no measurable peak in the chromatogram was found using the ND-SPME method described here, no freely dissolved PAHs could be quantified in the wastewater. Calibrations showed good linearity as indicated by the values of $r^{2}$. The total concentration varied from $3.96 \mu \mathrm{g} \mathrm{L}^{-1}$ for anthracene to $385 \mu \mathrm{g} \mathrm{L}^{-1}$ for fluorene. These results have further reinforced the hypothesis of the expected binding effect of the complex matrix. This method showed to be suitable for differentiating total PAH concentration from freely dissolved PAH in an oily wastewater. A drawback to a better understanding of the interactions between NOM and NAPL in the SPME technique was the lack of an extensive characterization of both NOM and NAPL in the wastewater. By determining the fractions of NOM and NAPL and their chemical composition, more conclusions could be withdrawn.

\section{Conclusions}

An efficient methodology was developed for the determination of both total and freely dissolved PAH in an oily wastewater. The use of ND-SPME for the free fraction and the use of an increase in temperature for a total PAH determination was successfully implemented for an oily wastewater sample. As explained earlier, the use of these two methods enables the differentiation between the freely dissolved and the bonded fractions of PAHs. The use of a model NOM and NAPL pointed out the interference of both components in total PAH determination. This interference was confirmed with the analysis of a real wastewater sample. The results showed that the matrix effect had such an interference that no PAH was detected when analyzing the freely dissolved fraction. A more detailed analysis regarding the NOM and NAPL composition of the studied wastewater would certainly help to better understand the interactions between those variables and the PAH uptake. However, this characterization is a complex and difficult process requiring an extensive and deep analytical knowledge and techniques.

\section{Recommendations and perspectives}

The type of PAH and the nature of the matrix are key factors in the chemical analysis of oily wastewaters, and the main components responsible for PAH binding should be identified. Indeed, the specific sampling times, chromatographic conditions, and matrix binding effects may vary and require a first assessment, as presented in this work. Furthermore, other parameters may be considered in order to determine the most effective conditions when analyzing total $\mathrm{PAH}$, namely salinity, $\mathrm{pH}$, and surfactant effects. Future research should include a comparative study with other standard analytical methods, together with a detailed characterization of the NOMs and NAPLs present in the wastewater, for a more complete discussion of the results obtained in this work and a better understanding of the processes dealing with the SPME analysis.

Acknowledgments The authors gratefully acknowledge the financial support awarded to Rui Gomes through the individual grant SFRH/BD/ $18816 / 2004$ and the project POCI/AMB/61044/2004 by the Foundation for Science and Technology (Portugal). They also would like to thank Anthony Danko for the language review of the paper.

\section{References}

Ai J (1997) Headspace solid phase microextraction. Dynamics and quantitative analysis before reaching a partition equilibrium. Anal Chem 69:3260-3266

Al Zarooni M, Elshorbagy W (2006) Characterization and assessment of Al Ruwais refinery wastewater. J Hazard Mater 136:398-405

Arthur CL, Pawliszyn J (1990) Solid phase microextraction with thermal desorption using fused silica optical fibers. Anal Chem 62:2145-2148

Arthur CL, Potter DW, Buchholz KD, Motlagh S, Pawliszyn J (1992) Solid-phase microextraction for the direct analysis of water: theory and practice. Lc Gc-Mag Sep Sci 10:656-661

Bartelt RJ, Zilkowski BW (1999) Nonequilbrium quantitation of volatiles in air streams by solid-phase microextraction. Anal Chem 71:92-101

Brigante M, Zanini G, Avena M (2008) On the dissolution kinetics of humic acid particles. Effect of monocarboxylic acids. Chemosphere 71:2076-2081 
Busetti F, Heitz A, Cuomo M, Badoer S, Traverso P (2006) Determination of sixteen polycyclic aromatic hydrocarbons in aqueous and solid samples from an Italian wastewater treatment plant. J Chromatogr A 1102:104-115

Doong R, Chang S, Sun Y (2000) Solid-Phase microextraction and headspace solid-phase microextraction for the determination of high molecular-weight polycyclic aromatic hydrocarbons in water and soil samples. J Chromatogr Sci 38:528-534

Doong R, Lin Y (2004) Characterization and distribution of polycyclic aromatic hydrocarbon contaminations in surface sediment and water from Gao-ping River, Taiwan. Water Res 38:1733-1744

Gorecki T, Pawliszyn J (1997) Effect of sample volume on quantitative analysis by solid-phase microextraction. 1 . Theoretical considerations. Analyst 122:1079-1086

Jones KD, Tiller CL (1999) Effect of solution chemistry on the extent of binding of phenanthrene by a soil humic acid: a comparison of dissolved and clay bound humic. Environ Sci Technol 33:580587

King AJ, Readman JW, Zhou JL (2003) The application of solid-phase micro-extraction (SPME) to the analysis of polycyclic aromatic hydrocarbons (PAHs). Environ Geochem Health 25:69-75

King AJ, Readman JW, Zhou JL (2004) Determination of polycyclic aromatic hydrocarbons in water by solid-phase microextractiongas chromatography-mass spectrometry. Anal Chim Acta 523:259-267

Kose T, Miyagishi A, Mukai T, Takimoto K, Okada M (2003) Effect of non-aqueous phase liquid on biodegradation of PAHs in spilled oil on tidal flat. Water Sci Technol 47(7-8):243-250

Kubicki JD, Apitz SE (1999) Models of natural organic matter and interactions with organic contaminants. Org Geochem 30:911-927

Lee P-H, Ong SK, Golchin J, Nelson GL (2001) Use of solvents to enhance PAH biodegradation of coal tar. Water Res 35:39413949

Lesage S, Brown S, Millar K, Novakowski K (2001) Humic acids enhanced removal of aromatic hydrocarbons from contaminated aquifers: developing a sustainable technology. J Environ Sci Health A36(8):1515-1533

Luan T, Fang S, Zhonga Y, Lina L, Chanb S, Lana C, Tamb N (2007) Determination of hydroxy metabolites of polycyclic aromatic hydrocarbons by fully automated solid-phase microextraction derivatization and gas chromatography-mass spectrometry. J Chromatogr A 1173:37-43

Macey RI, Oster GF (1993) Berkeley Madonna. http://www.berkeley madonna.com/index.html.

Maxin CR, Kogel-Knabner I (1995) Partitioning of polycyclic aromatic hydrocarbons (PAH) to water-soluble soil organic matter. Eur J Soil Sci 46:193-204

Mayer P, Vaes WHJ, Hermens JLM (2000) Absorption of hydrophobic compounds into the poly(dimethylsiloxane) coating of solidphase microextraction fibers: high partition coefficients and fluorescence microscopy images. Anal Chem 72:459-464

Ouyang G, Pawliszyn J (2006a) Recent developments in SPME for onsite analysis and monitoring. Trac-Trend Anal Chem 25:692-703
Ouyang G, Pawliszyn J (2006b) SPME in environmental analysis. Anal Bioanal Chem 386:1059-1073

Pawliszyn J (1997) Solid phase nicroextraction: theory and practice. Wiley-VCH

Poerschmann J, Kopinke F-D, Pawliszyn J (1998) Solid-phase microextraction for determining the binding state of organic pollutants in contaminated water rich in humic organic matter. $\mathrm{J}$ Chromatogr A 816:159-167

Poerschmann J, Kopinke FD (2001) Sorption of very hydrophobic organic compounds (VHOCs) on dissolved humic organic matter (DOM). 2. Measurement of sorption and application of a flory-huggins concept to interpret the data. Environ Sci Technol 35:1142-1148

Potter DW, Pawliszyn J (1994) Rapid determination of polyaromatic hydrocarbons and polychlorinated biphenyls in water using solidphase microextraction and GC/MS. Environ Sci Technol 28:298305

Ramos EU, Meijer SN, Vaes WHJ, Verhaar HJM, Hermens JLM (1998) Using solid-phase microextraction to determine partition coefficients to humic acids and bioavailable concentrations of hydrophobic chemicals. Environ Sci Technol 32:3430-3435

Rumble J, Lee AY, Blakeslee D, Young S (2001) Reliable solubility data in the age of computerized chemistry. Why, how, and when? Pure Appl Chem 73:825-829

Samanta SK, Singh OV, Jain RK (2002) Polycyclic aromatic hydrocarbons: environmental pollution and bioremediation. Trends Biotechnol 20:243-248

Schlautman MA, Morgan JJ (1993) Effects of aqueous chemistry on the binding of polycyclic aromatic hydrocarbons by dissolved humic materials. Environ Sci Technol 27:961-969

Shokrollahzadeh S, Azizmohseni F, Golmohammad F, Shokouhi H, Khademhaghighat F (2008) Biodegradation potential and bacterial diversity of a petrochemical wastewater treatment plant in Iran. Bioresource Technol 99:6127-6133

Vaes WHJ, Ramos EU, Verhaar HJM, Seinen W, Hermens JLM (1996) Measurement of the free concentration using solid-phase microextraction: binding to protein. Anal Chem 68:4463-4467

Van Hamme JD, Ward OP (2000) Development of a method for the application of solid-phase microextraction to monitor biodegradation of volatile hydrocarbons during bacterial growth on crude oil. J Ind Microbiol Biot 25:155-162

Wong H, Mok KM, Fan XJ (2007) Natural organic matter and formation of trihalomethanes in two water treatment processes. Desalination 210:44-51

Zhang Z, Pawliszyn J (1993a) Analysis of organic compounds in environmental samples by headspace solid phase microextraction. J High Res Chromatog 16:689-692

Zhang Z, Pawliszyn J (1993b) Headspace solid-phase microextraction. Anal Chem 65:1843-1852

Zhang Z, Pawliszyn J (1993c) Headspace analysis of soil samples using solid-phase microextraction. Abstr Pap Am Chem S 206:6ENVR

Zhu W, Tang X (2005) Choice of filters for treatment of oily wastewater from machine process. Ind Water Wastewater 36:78-80 